\title{
Automatic Substantia Nigra Segmentation in Neuromelanin-Sensitive MRI by Deep Neural Network in Patients With Prodromal and Manifest Synucleinopathy
}

\author{
R. KRUPIČKA ${ }^{1}$, S. MAREČEK ${ }^{2}$, C. MALÁ ${ }^{1}$, M. LANG ${ }^{1}$, O. KLEMPÍR ${ }^{1}$, T. DUSPIVOVÁ ${ }^{1}$, \\ R. ŠIROKÁ ${ }^{1}$, T. JAROŠÍKOVÁ ${ }^{1}$, J. KELLER ${ }^{2,3}$, K. ŠONKA ${ }^{2}$, E. RŮŽIČKA ${ }^{2}$, P. DUŠEK ${ }^{2}$ \\ ${ }^{1}$ Czech Technical University in Prague, Faculty of Biomedical Engineering, Kladno, Czech \\ Republic, ${ }^{2}$ Department of Neurology and Center of Clinical Neuroscience, First Faculty of \\ Medicine, Charles University and General University Hospital in Prague, Prague, Czech Republic, \\ ${ }^{3}$ Department of Radiodiagnostics, Na Homolce Hospital, Prague, Czech Republic
}

Received August 27, 2019

Accepted September 25, 2019

\begin{abstract}
Summary
Neuromelanin (NM) is a black pigment located in the brain in substantia nigra pars compacta (SN) and locus coeruleus. Its loss is directly connected to the loss of nerve cells in this part of the brain, which plays a role in Parkinson's Disease. Magnetic resonance imaging (MRI) is an ideal tool to monitor the amount of NM in the brain in vivo. The aim of the study was the development of tools and methodology for the quantification of NM in a special neuromelanin-sensitive MRI images. The first approach was done by creating regions of interest, corresponding to the anatomical position of SN based on an anatomical atlas and determining signal intensity threshold. By linking the anatomical and signal intensity information, we were able to segment the SN. As a second approach, the neural network U-Net was used for the segmentation of SN. Subsequently, the volume characterizing the amount of NM in the $\mathrm{SN}$ region was calculated. To verify the method and the assumptions, data available from various patient groups were correlated. The main benefit of this approach is the observer-independency of quantification and facilitation of the image processing process and subsequent quantification compared to the manual approach. It is ideal for automatic processing many image sets in one batch.
\end{abstract}

\section{Key words}

Neuromelanin segmentation - Deep neural network • U-net • REM sleep behavior disorder $\bullet$ Parkinson disease

\section{Corresponding author}

R. Krupička, Czech Technical University in Prague, Faculty of Biomedical Engineering, nam. Sitna 3105, Kladno, Czech Republic. E-mail: krupicka@fbmi.cvut.cz

\section{Introduction}

Neuromelanin is a complex black pigment composed of melanin, metal ions, lipids, and proteins present in dopamine-producing neurons in the substantia nigra (SN) (Zucca et al. 2017). In neurodegenerative disorders from the synucleinopathy group, neurons containing neuromelanin are typically at a higher risk of cell death than neurons without pigmentation. The death of these neurons leads to the depletion of dopamine in the corpus striatum ultimately causing parkinsonian symptoms, i.e. akinesia, tremor, and rigidity that are typical for Parkinson's disease (PD) (Zecca et al. 2001, Haining and Achat-Mendes 2017). Since there is a functional reserve in the number of dopaminergic neurons, parkinsonism appears only when approximately $50 \%$ of neurons are lost.

Nowadays, synucleinopathy may be diagnosed in the prodromal stage, i.e. prior to the development of parkinsonism (Berg et al. 2015). The strongest marker of prodromal synucleinopathy is REM sleep behaviour disorder (RBD) presenting as loss of muscle atonia during REM sleep and abnormal behavior during sleep (Montplaisir 2004). Long term studies have proven that $70-90 \%$ of people suffering from RBD develop PD or another neurodegenerative disease, such as dementia with Lewy bodies or multiple system atrophy, in 12 and more years (Postuma et al. 2019, Kim et al. 2017).

Pigmentation of the substantia nigra (SN) with 
neuromelanin is a physiological process inasmuch as it is a byproduct of oxidative catabolism of catecholamines. The volume of pigmented neurons, which is increasing during life until approximately the age of 60 , typically decreases thereafter. The loss of neuromelanin in $\mathrm{SN}$ is directly connected to the degeneration of dopaminergic neurons in SN and suggests PD or other neurodegenerative diseases. (Zecca et al. 2001)

Neuromelanin-sensitive imaging is a promising MR technique for the assessment of SN integrity. The contrast mechanism of neuromelanin is based on its very short $\mathrm{T} 1$ relaxation time (Trujillo et al. 2017). This approach may be beneficial for detecting and monitoring of degeneration in prodromal stages of synucleinopathy, e.g. in patients with RBD. Another method used for such purposes is dopamine transporter (DAT) imaging using scintigraphy. However, these methods are not suitable for longitudinal monitoring because they are associated with radiation exposure and high costs (Ohtsuka et al. 2014).

Several approaches are currently used for the processing of neuromelanin-sensitive MRI scans. They typically involve segmentation based on thresholding voxels in the $\mathrm{SN}$ regions relative to the surrounding tissue (Langley et al. 2015, Castellanos et al. 2015, Schwarz et al. 2011). All of them are at least partly done manually. This human factor is the biggest problem in case of processing big data or creating a reliable, precise and unbiased quantification system.

Our work was therefore focused on automatization of data processing and elimination of the human factor during the evaluation of the data acquired from neuromelanin-sensitive MRI. This should help to process more data in a shorter time and increase the precision and reliability of obtained results. To maintain the possibility to process even non-standard data the intervention of reading physician to the whole automatic process was kept.

\section{Patients and Methods}

\section{Participants}

In total, there were 122 subjects involved in the study. 36 (8F, 28M) with PD, mean age $60.1 \pm 9.4 \mathrm{yrs}, 47$ $(5 \mathrm{~F}, 42 \mathrm{M})$ with $\mathrm{RBD}$, mean age $67.9 \pm 4.4 \mathrm{yrs}$, and 39 (5F, 34M) healthy controls (HC), mean age $65.0 \pm$ 6.7 yrs. All PD patients were treatment-naïve and diagnosed in accordance with the MDS clinical diagnostic criteria. The RBD diagnosis was confirmed by video-polysomnography according to the International
Classification of Sleep Disorders, third edition (ICSD-3) (American Academy of Sleep Medicine, 2014). For inclusion, RBD patients had to be $>49$ years, and to be without overt parkinsonism, dementia, as well as factors indicative of secondary RBD such as narcolepsy, druginduced RBD (i.e. RBD originating shortly after initiation of antidepressants), or focal brainstem lesions on MRI. Control subjects were recruited from the general community through advertisements. To be eligible for the study, controls had to be free of major neurologic disorders, active oncologic illness, and abuse of psychoactive substances. In all control subjects, RBD was excluded by thorough history and videopolysomnography.

\section{MRI scanning protocol}

Patients were examined on a 3T whole-body MRI system (Skyra, Siemens Healthcare, Erlangen, Germany) equipped with 32-channel head coil. The neuroimaging protocol included: 3D-Magnetization Prepared - Rapid Gradient Echo: (MPRAGE; 176 axial slices covering entire brain, repetition time $[\mathrm{TR}]=2000$ $\mathrm{ms}$, echo time $[\mathrm{TE}]=2.4 \mathrm{~ms}$, inversion time $[\mathrm{TI}]=900 \mathrm{~ms}$, spatial resolution $=1 \times 1 \times 1 \mathrm{~mm}$ ) and neuromelaninsensitive (NM) 2D-gradient echo with magnetization transfer (MT) preparation prepulse (14 axial slices perpendicular to dorsal margin of the brainstem covering entire $\mathrm{SN}, \mathrm{TR}=394 \mathrm{~ms}, \mathrm{TE}=2.7 \mathrm{~ms}$, spatial resolution $=0.5 \times 0.5 \times 3 \mathrm{~mm}$, number of measurements $=7$ ) sequences.

\section{Software}

For processing of MRI images and for deep learning implementation, we used MATLAB. In addition, we used SPM 12 (Statistical Parametric Mapping) (Friston 2007) for image preprocessing and LeadDBS toolbox (Horn and Kühn 2015) for acquiring probability maps.

\section{Data processing}

All patient data obtained from the Department of Neurology were anonymized. Imaging data were acquired from Na Homolce Hospital.

\section{Pre-processing of images}

First, NM images acquired by seven measurements were realigned and averaged for each patient.

The second step of processing was co- 
registration of MPRAGE images to the NM image using SPM12. Mutual registration of sequences enabled us to localize anatomical information contained in NM images in topographical MPRAGE images of the entire brain. The output was a transformation matrix, which would be applied to images in the last step of the process.

The next step was brain tissue segmentation and the spatial normalization to the standard MNI space. The segmentation was done on the MPRAGE image based on TPM_Lorio_Draganski.nii template (Lorio et al. 2016) from LeadDBS.

The final step of image pre-processing was definition of the $\mathrm{SN}$ region of interest (ROI) in the standard space and its transformation to individual NM space of each subject. Previously, spatial normalization provided forward and back transformation matrices. SN area defined in MNI PD25 atlas (Xiao et al. 2017) was chosen and transformed into the NM images using the inverse transformation matrix.

\section{Substantia nigra segmentation}

Because of the inter-individual differences in SN shape and the number of slices displaying $\mathrm{SN}$, three inferior slices with neuromelanin signal were chosen for analysis.

\section{Threshold segmentation}

After applying the spatial prior based on the probability SN mask, NM volume was calculated by implementing the threshold of MRI signal in the area. For every patient, a reference region (circle with $4 \mathrm{~mm}$ diameter) was placed in the brainstem tissue of the inferior slice, outside of the SN signal. The mean signal value from this reference region multiplied by 1.2 was implemented as a signal threshold (Fig. 1). The constant 1.2 was defined empirically from images by the neurologist. Voxels with signal values above this threshold in the SN spatial prior were segmented. Voxels with signal above the threshold, which apparently did not belong to $\mathrm{SN}$, were manually cleaned using the MatrixUser v2.2 software. The resulting binary map of $\mathrm{SN}$ constituted the NM volume.

\section{Implementation of $U$-Net}

The segmented volume of NM from all subjects was used as an output for the training of the artificial convolutional neural network U-Net (Ronneberger et al. 2015). This neural network is optimized for use in the segmentation of biomedical images, so it does not need as

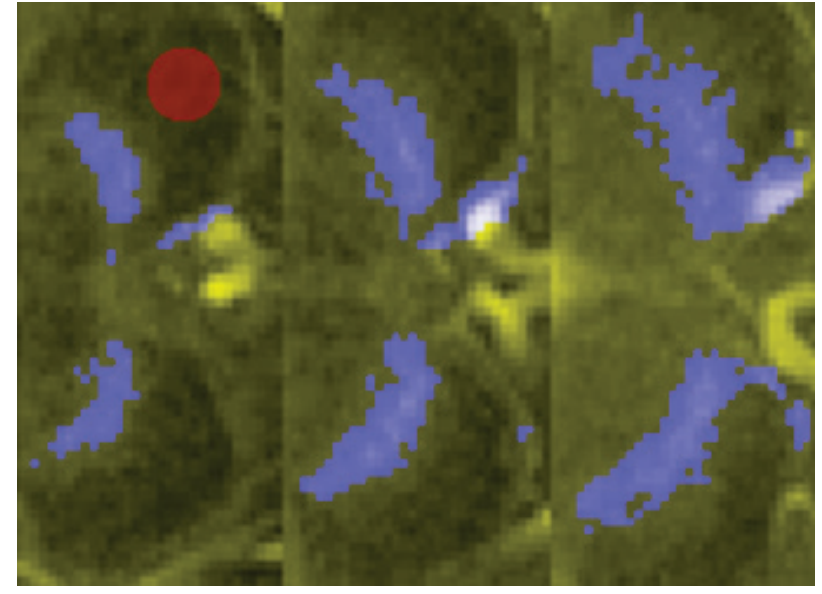

Fig. 1. Signal intensity above threshold (blue) in neuromelaninsensitive MRI image in the SN area and reference region (red circle).

many learning and training data as a standard convolutional neural networks.

For training of the neural network, a set of middle NM slices and corresponding segmented binary maps were used. We employed the "adam" method to train the models. The global learning rate was set to 0.001 and the batch size was set to 10 . In our pilot experiment, we arbitrarily tested several hyperparameters and then fixed it to 0.001 as it ensures the training could converge. We also performed a grid search afterward and tested as $0.01,0.005,0.003,0.001,0.0005$. We found that changes in this parameter did not alter the main conclusions in this paper. After 1000 epochs the precision of signal segmentation was approximately $99 \%$. Model learnt on the middle slice was used also for the segmentation of the superior and inferior slices. As a result of this process, a set of segmented maps of the NM signal was obtained (Fig. 2).

\section{Neuromelanin volume calculation and statistics}

We calculated the volume of NM from the threshold-segmented binary map of the $\mathrm{SN}$ area and from the automatically obtained probability map of SN area from U-net. We assumed that all labelled voxels in binary maps had a $100 \%$ probability. From the voxel size multiplied by the probability we obtained the volume for each slice and the total volume for all three slices of the $\mathrm{SN}$ area in $\mathrm{mm}^{3}$.

The NM volumes were computed for RBD, PD and $\mathrm{HC}$ groups. Normal distribution was confirmed by the Shapiro-Wilk test. HC was compared with PD and RBD using the t-test. 


\section{Results}

Calculated volumes of neuromelanin were subjected to statistical analysis to compare the ability of both segmentation methods to distinguish between PD, RBD and $\mathrm{HC}$ (see Table 1). It turned out that for signal threshold-segmentation there was only a significant difference between RBD group and $\mathrm{HC}$ in the superior slice $(p=0.04)$.

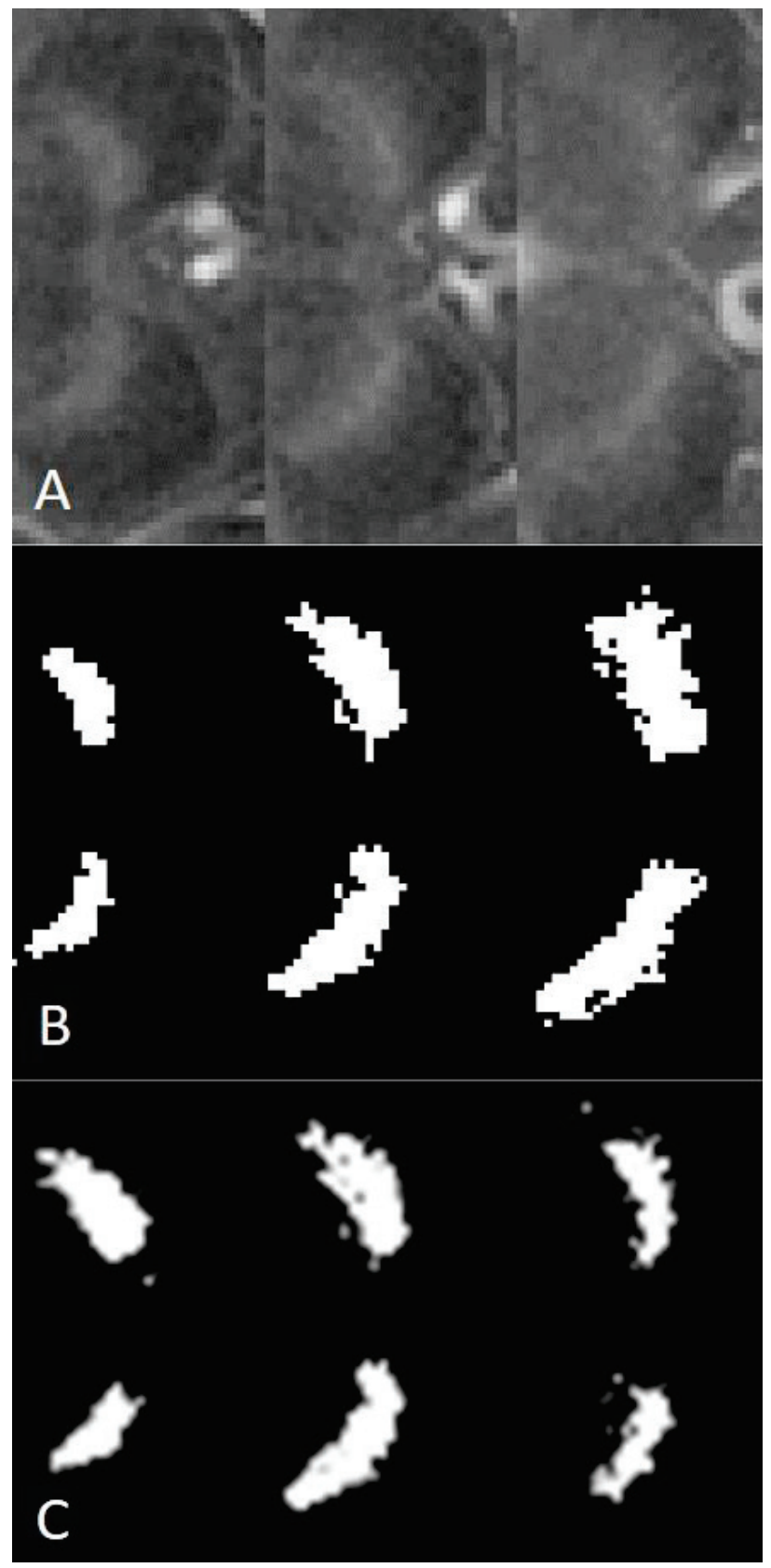

Fig. 2. Results of segmentation. Neuromelanin-sensitive MRI image (A), corresponding segmentation image using anatomical localisation and signal intensity threshold (B) and using artificial convolutional neural network U-Net (C).
On the other hand, segmentation using U-Net distinguished between $\mathrm{HC}$ and $\mathrm{RBD}$ patient groups in superior slices $(\mathrm{p}=0.027)$ and $\mathrm{HC}$ and $\mathrm{PD}$ patients in superior $(p=0.001)$, middle slices $(p=0.017)$ and in the whole volume $(\mathrm{p}=0.013)$.

Table 1. Differences in the RBD and PD groups compared to healthy controls $(\mathrm{HC})$, SN volumes segmented by threshing and U-net segmentation.

\begin{tabular}{|c|c|c|c|c|}
\hline & \multicolumn{2}{|c|}{$\begin{array}{c}\text { Threshold } \\
\text { segmentation }\end{array}$} & \multicolumn{2}{|c|}{$\begin{array}{c}\text { U-net } \\
\text { segmentation }\end{array}$} \\
\hline & $\begin{array}{c}\text { mean } \\
\pm \mathbf{s d} \\
{\left[\mathbf{m m}^{3}\right]}\end{array}$ & $\begin{array}{c}\text { t-test } \\
p\end{array}$ & $\begin{array}{c}\text { mean } \\
\pm \mathbf{s d} \\
{\left[\mathbf{m m}^{3}\right]}\end{array}$ & $\begin{array}{c}\text { t-test } \\
\text { p }\end{array}$ \\
\hline \multicolumn{5}{|c|}{ Superior slice } \\
\hline$H C$ & $336 \pm 67$ & & $71 \pm 45$ & \\
\hline$R B D$ & $300 \pm 72$ & 0.040 & $52 \pm 34$ & 0.027 \\
\hline$P D$ & $300 \pm 95$ & 0.060 & $40 \pm 36$ & 0.001 \\
\hline \multicolumn{5}{|c|}{ Middle slice } \\
\hline$H C$ & $234 \pm 46$ & & $123 \pm 53$ & \\
\hline$R B D$ & $227 \pm 46$ & 0.449 & $117 \pm 48$ & 0.551 \\
\hline$P D$ & $218 \pm 67$ & 0.213 & $94 \pm 50$ & 0.017 \\
\hline \multicolumn{5}{|c|}{ Inferior slice } \\
\hline$H C$ & $45 \pm 39$ & & $31 \pm 26$ & \\
\hline \multirow[t]{2}{*}{$R B D$} & $57 \pm 39$ & 0.178 & $38 \pm 28$ & 0.284 \\
\hline & $52 \pm 39$ & 0.477 & $32 \pm 24$ & 0.859 \\
\hline \multicolumn{5}{|c|}{ All slices } \\
\hline$H C$ & $615 \pm 136$ & & $225 \pm 113$ & \\
\hline$R B D$ & $584 \pm 133$ & 0.289 & $207 \pm 88$ & 0.410 \\
\hline$P D$ & $570 \pm 229$ & 0.299 & $166 \pm 86$ & 0.013 \\
\hline
\end{tabular}

\section{Discussion}

In this study we have shown superior performance of U-net compared to manual thresholdedsegmentation in discriminating controls from patients with prodromal and manifest synucleinopathy.

Overall, the results of the quantification were not as promising as we hoped for. We assumed the biggest difference between control and PD patients in the caudate slice since SN neurodegeneration begins in its inferior part; this was, however, not confirmed. On the contrary, superior slices segmented by the neural network U-Net 
showed the best discriminative power for differentiation of HC, RBD and PD groups. Results in the superior slice correspond to previously published values in RBD and PD in the literature (Schwarz et al. 2011, Castellanos et al. 2015, Isaias et al. 2016, Trujillo et al. 2017). The pattern of mean NM volumes in this slice, i.e. $\mathrm{HC}>\mathrm{RBD}>\mathrm{PD}$ correspond with the theory that the volume of neuromelanin gradually decreases from prodromal to manifest synucleinopathy.

The variance of calculated values was quite large, especially in threshold-segmentation, PD group, and in the inferior slice. This could be caused by the relatively large slice thickness that was $3 \mathrm{~mm}$. In the inferior slices, $\mathrm{SN}$ could be not present in the entire slice volume leading to partial volume effect. This is the main limit of the pipeline that warrants further technical refinement in the future. The variance could be also contributed by the large age dispersion in patient groups, various stages of the disease and less precise segmentation. Results from U-Net, on the other hand, showed a smaller standard deviation and appeared to be more stable and accurate. A larger set of patients would be needed to prove the functionality of this method with at least 100 patients in every group.

Our biggest neuroscience engineering contribution lies in the usage of the U-Net segmentation, that opens up possibilities in segmenting of the neuromelanin in the brain. Secondly, the whole pipeline allows applying a broad spectrum of analytical steps to neuromelanin-sensitive data in a fully automated, largescale fashion, which brings significant research value to the consumer compared to other approaches used for neuromelanin quantification, which require manual input (Schwarz et al. 2011, Castellanos et al. 2015, Isaias et al. 2016, Trujillo et al. 2017).

\section{Conclusion}

In this project, we created the pipeline processing raw image datasets from clinical MRI. This pipeline can localize substantia nigra, perform segmentation and calculate the volume of neuromelanin in neuromelanin- sensitive MRI images. This process uses two different approaches in neuromelanin signal segmentation. The first one is more traditional, with anatomical atlas-based localization combined with signal intensity threshold. The results of this method were not perfectly accurate but could be used as training data for the artificial convolutional neural networks.

The second approach in signal segmentation is artificial convolutional neural network U-Net. Using this neural network, segmentation results are more accurate and better correspond to the theoretical assumptions about the volume of neuromelanin in the brain in different stages of synucleinopathies.

\section{Conflict of Interest}

There is no conflict of interest.

\section{Acknowledgements}

This study was supported by the Czech Science Foundation, GACR 16-07879S, and Czech Ministry of Health, AZV 15-25602A and DRO (NHH, 00023884) and Progres Q27/1LF.

\section{References}

AMERICAN ACADEMY OF SLEEP MEDICINE. International classification of sleep disorders. 3rd ed. Darien, IL: American Academy of Sleep Medicine, 2014.

BERG D, POSTUMA RB, ADLER CH, BLOEM BR, CHAN P, DUBOIS B, GASSER T, GOETZ CG, HALLIDAY G, JOSEPH L, LANG AE10, LIEPELT-SCARFONE I, LITVAN I, MAREK K, OBESO J13, OERTEL W, OLANOW CW, POEWE W, STERN M, DEUSCHL G: MDS research criteria for prodromal Parkinson's disease. Mov Disord 30: 1600-1611, 2015.

CASTELLANOS G, FERNÁNDEZ-SEARA MA, LORENZO-BETANCOR O, ORTEGA-CUBERO S, PUIGVERT M, URANGA J, VIDORRETA M, IRIGOYEN J, LORENZO E, MUÑOZ-BARRUTIA A, ORTIZ-DESOLORZANO C, PASTOR P, PASTOR MA: Automated neuromelanin imaging as a diagnostic biomarker for Parkinson's disease. Mov Disord 30: 945-952, 2015.

FRISTON KJ: Statistical parametric mapping: the analysis of funtional brain images. Boston: Elsevier/Academic Press, 2007. ISBN 9780123725608.

HAINING B, ACHAT-MENDES C: Neuromelanin, one of the most overlooked molecules in modern medicine, is not a spectator. Neural Regen Res 12: 372-375, 2017. 
HORN A, KÜHN A: Lead-DBS: a toolbox for deep brain stimulation electrode localizations and visualizations. Neuroimage 107: 127-135, 2015.

ISAIAS I, TRUJILlO P, SUMMERS PE, MAROTTA G, MAINARDI L, PEZZOLI G, ZECCA L, COSTA A: Neuromelanin imaging and dopaminergic loss in Parkinson's disease. Front Aging Neurosci 8: 196, 2016.

LANGLEY J, HUDDLESTON DE, CHEN X, SEDLACIK J, ZACHARIAH N, HU X: A multicontrast approach for comprehensive imaging of substantia nigra. Neuro Image 112: 7-13, 2015.

KIM Y, KIM Y E, PARK E O, SHIN CW, KIM HJ, JEON B: REM sleep behavior disorder portends poor prognosis in Parkinson's disease: A systematic review. J Clin Neurosci 47: 6-13, 2018.

LORIO S, FRESARD S, ADASZEWSKI S, Kherif F, CHOWDHURY R, FRACKOWIAK RS, ASHBURNER J, HELMS G, WEISKOPF N, LUTTI A, DRAGANSKI B: New tissue priors for improved automated classification of subcortical brain structures on MRI. Neuroimage 130: 157-166, 2016.

LORIO S, FRESARD S, ADASZEWSKI S, KHERIF F, CHOWDHURY R, FRACKOWIAK RS, ASHBURNER J, HELMS G, WEISKOPF N, LUTTI A, DRAGANSKI B: New tissue priors for improved automated classification of subcortical brain structures on MRI. Neuroimage 130: 157-166, 2016.

MONTPLAISIR J: Abnormal motor behavior during sleep. Sleep Medicine 5: 34-34, 2004.

OHTSUKA C, SASAKI M, KONNO K, KATO K, TAKAHASHI J, YAMASHITA F, TERAYAMA Y: Differentiation of early-stage Parkinsonisms using neuromelanin-sensitive magnetic resonance imaging. Parkinsonism Relat Disord 20: 755-760, 2014.

POSTUMA RB, IRANZO A, HU M, HÖGL B, BOEVE BF, MANNI R, OERTEL WH, ARNULF I, FERINISTRAMBI L, PULIGHEDDU M, ANTELMI E, COCHEN DE COCK V, ARNALDI D, MOLLENHAUER B, VIDENOVIC A, SONKA K, JUNG KY, KUNZ D, DAUVILlIERS Y, PROVINI F, LEWIS SJ, BUSKOVA J, PAVLOVA M, et al.: Risk and predictors of dementia and parkinsonism in idiopathic REM sleep behaviour disorder: a multicentre study. Brain 142: 744-759, 2019.

RONNEBERGER O, FISCHER P, BROX T: "U-Net: Convolutional Networks for Biomedical Image Segmentation". arXiv:1505.04597, 2015.

SCHWARZ ST, RITTMAN T, GONTU V, MORGAN PS, BAJAJ N, AUER DP: T1-weighted MRI shows stagedependent substantia nigra signal loss in Parkinson's disease. Movement Disorders 26: 1633-1638, 2011.

TRUJILLO P, SUMMERS PE, FERRARI E, ZUCCA FA, STURINI M, MAINARDI LT, CERUTTI S, SMITH AK, SMITH SA, ZECCA L, COSTA A: Contrast mechanisms associated with neuromelanin-MRI. Magn Reson Med 78: 1790-1800, 2017.

XIAO Y, FONOV V, CHAKRAVARTY MM, BÉRIAULT S, AL-SUBAIE F, SADIKOT A, PIKE GB, BERTRAND G, COLLINS DL: A dataset of multi-contrast population-averaged brain MRI atlases of a Parkinson's disease cohort. NeuroImage 12: 370-379, 2017.

ZECCA L, TAMPELLINI D, GERLACH M, RIEDERER P, FARIELLO R, SULZER D: Substantia nigra neuromelanin: structure, synthesis, and molecular behaviour. Mol Pathol 54: 414-418, 2001.

ZUCCA FA, SEGURA-AGUILAR J, FERRARI E, MUNOZ P, PARIS I, SULZER D, SARNA T, CASELLA L, ZECCA L: Interactions of iron, dopamine and neuromelanin pathways in brain aging and Parkinson's disease. Prog Neurobiol 155: 96-119, 2017. 